\title{
Desigualdades regionales y sociales del rendimiento escolar al término de la educación primaria en el Perú
}

\author{
Luis Muelle \\ Consultor en Educación \\ luismuelle@yahoo.com
}

Recibido: 15-05-2018

Aprobado: 03-02-2019 


\title{
Desigualdades regionales y sociales del rendimiento escolar al término de la educación primaria en el Perú
}

\begin{abstract}
Resumen
La evaluación de aprendizajes aplicada en la Encuesta Muestral 2013 a los alumnos de sexto grado de primaria en las competencias de Lectura, Matemática y Ciudadanía permite conocer la dimensión de las desigualdades en los rendimientos académicos, en particular, a nivel regional, así como la influencia que ejercen su perfil socioeconómico y la composición social de las escuelas a las que asiste. Los resultados evidencian que el mayor rendimiento escolar se asocia positivamente con la condición socioeconómica del alumno, quien se benefician relativamente más si ya posee una mejor posición social. El vínculo entre rendimiento escolar y condición socioeconómica se observa de manera más explícita en la composición social de las escuelas: los alumnos socialmente favorecidos asisten a escuelas favorecidas y obtienen siempre mejores rendimientos en todas las competencias.

A nivel regional, el impacto social sobre el rendimiento es distinto según cada región y cada competencia, y se constata que la ventaja en los logros académicos de la escuela privada sobre la pública persiste y alcanza brechas importantes. También, se observa que hay regiones socialmente más igualitarias con menor rendimiento, así como regiones menos igualitarias, pero con mayor rendimiento. Con diferentes magnitudes, existen diferencias al interior de las regiones y entre regiones, en la medida que estas atienden tanto alumnos como escuelas socialmente diferentes.

Ciertamente, un sistema educativo resulta más eficiente y equitativo cuanto mayor sean sus logros y menor sea la influencia de las condiciones sociales del alumno y de la escuela, pero la dimensión de estas desigualdades en las regiones del país reclama intervenciones de políticas educativas urgentes desde la educación primaria.
\end{abstract}

Palabras clave: Capital humano, rendimiento escolar, desigualdad regional, composición social 


\title{
Regional and social inequalities in student achievement at the last grade of primary education in Peru
}

\begin{abstract}
The 2013 Survey Sample assessment in Reading, Mathematics and Citizenship competences applied to students at the end of Peruvian primary school cycle shows the influence of the student socio-economic profile and the school social composition, as well as the inequalities of achievement, particularly, at the regional level. The results evidence that the highest school performance is positively associated with the socioeconomic condition of the student, who benefits relatively more if he/she already belongs to high socioeconomic class. The connection between school performance and socioeconomic condition influences the school's social composition: socially privileged students attend privileged schools and always obtain better performances in all the academic competences.

At the regional level, the social impact on the performance of students is different according to each region and to each competition. The advantage in performance of private schools over public schools persists: between the former and the latter, there are important gaps. Moreover, there are socially more egalitarian regions with lower performance, but also less egalitarian regions with higher performance. With different magnitudes, these differences occur within and between regions to the extent that students and school are also socially different.

There is a consensus that an education system is more efficient and equitable according to its achievements and the lesser the influence of the social status of the student. Nonetheless, the persistence of social inequalities on achievements in the country's regions calls for urgent educational policy action, specially, in the case of primary schools.
\end{abstract}

Keywords: Human capital, student achievement, regional inequality, social composition 


\section{Introducción}

Desde hace tiempo, la investigación en educación intenta identificar en qué medida los logros del aprendizaje obedecen a la influencia de los factores sociales asociados a los procesos educativos. Dentro de este campo de estudio, la desigualdad social frente a la escuela se convierte en un tema importante a considerar en la implementación de toda política de educación. Ciertamente, el país ha realizado progresos en el acceso al sistema escolar - en particular, la educación primaria-, pero la desigualdad de los rendimientos de los alumnos persiste cuando estas se asocian a aspectos como la localización geográfica, la gestión de la escuela, el género, la lengua, entre otros. Cada vez, hay un mayor consenso acerca de cuáles son los factores importantes que originan estas desigualdades, tales como el contexto social del alumno y la composición social de las escuelas.

En el Perú, las evaluaciones de alumnos, a cargo de la Unidad de Medición de la Calidad de los Aprendizajes (UMC) del Ministerio de Educación, tienen un carácter tanto censal como muestral. Estas evaluaciones se aplican en grados y niveles representativos a escala nacional. La Evaluación Censal de Estudiantes (ECE) de segundo y cuarto grado de primaria (Lectura y Matemáticas) y segundo grado de secundaria (Lectura, Matemáticas, Historia/Geografía/ Economía) se ejecuta desde el 2007. También, se ha realizado evaluaciones muestrales en los años 1996, 1998, 2001 y 2004. La última evaluación muestral con datos disponible a la fecha de este estudio es la del 2013.

En cuanto a las regiones, el sistema educativo nacional tiene un carácter descentralizado. Las Direcciones Regionales de Educación (DRE) cuentan con importantes niveles de autonomía frente al Gobierno nacional y, por lo mismo, el impacto de su gestión resulta importante.

Las evaluaciones muestrales contienen información más detallada sobre aspectos sociales de alumnos y escuelas que las evaluaciones censales, lo cual favorece un análisis más minucioso sobre los factores sociales que encuadran los aprendizajes. Por este motivo, para los propósitos de este trabajo, se utilizará la base de datos, que será denominada UMC-EM 2013. Esta evaluación tuvo como objetivos principales informar acerca de los logros de aprendizaje de alumnos en sexto grado de primaria en las competencias de lectura, escritura, matemática y ciudadanía. La decisión de conducir una evaluación en sexto grado apunta a identificar en qué medida los alumnos que están próximos a concluir el quinto ciclo de la Educación Básica Regular (EBR) - final del ciclo - han desarrollado las capacidades establecidas en el currículo nacional. De este modo, es posible facilitar las decisiones de política para mejorar la calidad de los aprendizajes.

Este trabajo pretende favorecer la implementación de políticas de educación aportando respuestas a las cuestiones sobre las desigualdades sociales en la escuela, a partir de los resultados de los aprendizajes a nivel de la región. En 
función de ello, en primer lugar, el estudio propone analizar la influencia de la condición socioeconómica de los alumnos y de las escuelas sobre las desigualdades del rendimiento académico en el último grado de educación primaria y en cada región del país para apreciar la magnitud de su impacto. En segundo lugar, busca contribuir a incorporar la dimensión regional en la discusión sobre la desigualdad y la equidad del rendimiento. El propósito es caracterizar las brechas existentes entre las regiones de administración del sistema de educacional nacional.

En la siguiente sección, se presenta la literatura aferente a los propósitos de este estudio. Luego, se desarrolla el marco metodológico que preside la selección de variables y métodos que permiten asociar los perfiles sociales de alumnos y escuelas con el desempeño académico. Luego, se muestran los resultados del análisis con énfasis en la diversidad regional, según cada una de las tres competencias evaluadas por la UMC-EM 2013. Finalmente, se plantean las conclusiones y las reflexiones que pretenden contribuir con la construcción de políticas educativas.

\section{Revisión de literatura}

Una breve revisión de la literatura sobre los condicionantes sociales del rendimiento académico tanto en el ámbito nacional como internacional da cuenta de la abundante disponibilidad de información desde la época de Coleman et al. (1966) y Bourdieu (1970). En efecto, múltiples estudios se han dedicado a demostrar la influencia preponderante de los antecedentes sociales de los alumnos sobre las desigualdades en el acceso, la permanencia y el rendimiento en todos los niveles del sistema educativo.

La conocida y extensa revisión metanalítica efectuada por Sirin (2005) sobre la literatura académica publicada entre los años 1990 y 2000 destaca la abundancia de trabajos que abordan el vínculo entre la condición social del alumno y el logro académico. Rumberger y Palardy (2005), por su parte, identifican que la composición social de la escuela ejerce una influencia mayor en el rendimiento académico que el origen social alumno. A este factor se suman las expectativas docentes, las tareas escolares, la cobertura curricular, el clima escolar y el tipo de gestión de las escuelas. Otros estudios confirman la importancia del origen social del alumnado que escolariza cada tipo de escuelas y que configura su composición socioeconómica (Calero, Escardíbul, Waisgrais y Mediavilla, 2007; Monseur y Crahay, 2008).

Desde una perspectiva económica, Hanushek y Woessmann (2011) destacan la literatura económica emergente que incorpora los resultados de las pruebas nacionales internacionales de logro educativo para analizar los determinantes del impacto económico de las competencias cognitivas de jóvenes y adultos, y comprobar así los efectos del capital humano en la distribución del ingreso y el crecimiento económico de un país. 
A nivel internacional, es importante la encuesta Terce (Unesco), que evaluó a los alumnos de tercero y sexto grado en 15 países de América Latina ${ }^{1}$ en el año 2013. En sexto grado, fueron evaluadas las competencias de Lectura, Matemática y Ciencias. En el Perú, se evaluó a aproximadamente 9950 estudiantes de 297 escuelas; además, se aplicó cuestionarios a los estudiantes, docentes, directores y padres de familia ${ }^{2}$. El informe de este estudio concluye que el desempeño de los alumnos de la región latinoamericana está principalmente influenciado por sus características sociales. La magnitud y la fuerza de la relación existente entre logros académicos y nivel socioeconómico son los factores que visibilizan las amplias desigualdades de resultados. Estos resultados internacionales se alinean con los resultados nacionales, como se desarrollará adelante.

En el Perú, Cueto, León y Miranda (2014) dan cuenta de un vacío en la literatura sobre la diferenciación social de las escuelas y destacan el trabajo de Benavides, León y Etesse (2014), quienes, con datos de las encuestas Pisa 2000 y 2009, observan un cambio en la segregación escolar en las escuelas de secundaria. Los autores comprueban que, en ese período, dicha brecha se ha incrementado en el país, a diferencia de Argentina, Chile y Brasil, donde se ha reducido o mantenido (Benavides, León y Etesse, 2014).

Asimismo, los trabajos de Miranda (2008), Cueto (2007), Guadalupe y Castillo (2014), y Muelle (2016) en el Perú, con diferentes enfoques y métodos, destacan los diversos grados de influencia de los factores sociales. En ese marco, vale mencionar el balance de estudios realizado por León y Collahua (2016), quienes presentan un metanálisis que abarca los años 2000 a 2014. Los autores identifican 28 estudios que muestran el mayor efecto de la composición socioeconómica de la escuela sobre el logro con respecto al propio nivel socioeconómico del alumno y sugieren integrar esta composición como aspecto insoslayable de los factores asociados al logro. También, León y Youn (2016), en sus análisis del modelo multinivel sobre el rendimiento de alumnos, resaltan la presencia significativa de las variables que se refieren a procesos escolares, tales como el clima disciplinario en clase y el sentido de pertenencia a la escuela.

En cuanto a las regiones, resalta notablemente el estudio de Guadalupe y Castillo (2014) sobre las disparidades educativas aproximadas a través de la Evaluación Censal de Estudiantes. Esta investigación considera importantes los aspectos vinculados con (a) las disparidades socioeconómicas entre regiones, (b) el acompañamiento y supervisión educativos, (c) los patrones de asentamiento poblacional y costos asociados al desplazamiento de personas, (d) el acceso a la educación inicial, y (e) aspectos de gestión vinculados tanto con

1. La encuesta se aplicó en Argentina, Brasil, Chile, Colombia, Costa Rica, Ecuador, Guatemala, Honduras, México, Nicaragua, Panamá, Paraguay, Perú, República Dominicana y Uruguay, más el Estado de Nuevo León (México). 
los directores de escuela como con las políticas de incentivos implementadas por algunas Direcciones Regionales. Asimismo, para analizar los desempeños estudiantiles de manera contextualizada, estos son abordados con temas como la distribución regional del PIB, los Proyectos Educativos Regionales (PER), el índice de necesidad básica insatisfecha (NBI), el gasto público por alumno en primaria, entre otros.

Para enmarcar el presente estudio, es preciso presentar brevemente los propios resultados de la UMC-EM 2013. En efecto, el respectivo informe técnico (UMC, 2014) y el informe oficial (UMC, 2016) ${ }^{3}$ presentan y discuten in extenso los resultados obtenidos y los métodos analíticos aplicados. El segundo plantea la relación entre los puntajes obtenidos en las competencias y dicho rendimiento con el índice socioeconómico, según el tipo de gestión (estatal/ no estatal) y el área (urbana/rural) de la escuela (que es denominada como institución educativa o IE). En suma, establece la relación entre el rendimiento inicial y final de la primaria en el tiempo.

Con el propósito de incorporar otras características socioeconómicas de los alumnos, sus familias y las escuelas a las que pertenecen, se complementa el análisis teniendo en cuenta las características de los principales actores educativos (director, docente, estudiante y padres de familia). Dichas características han sido recogidas mediante cuestionarios de factores asociados. A partir de ello, se presenta una serie de modelos de factores asociados, que resultaron ser estadísticamente significativos para explicar las diferencias en el rendimiento con el uso del análisis multinivel.

$\mathrm{Al}$ respecto, conviene registrar la siguiente afirmación del informe:

Finalmente, a modo de resumen, se puede señalar que los resultados dan evidencias muy claras, en todos los casos, de que solo una pequeña proporción de estudiantes a nivel nacional logra los aprendizajes esperados para el final de la primaria. En todas las competencias evaluadas, se repite un patrón similar en la distribución de los resultados: quienes obtienen los resultados más bajos son los estudiantes que tienen una lengua originaria, que estudian en escuelas rurales fundamentalmente multigrado, y que viven en regiones ubicadas en la sierra sur y en la selva del país. Es decir, estos resultados tienen una asociación significativa con los antecedentes sociales, culturales y económicos de los hogares de los estudiantes evaluados (UMC, 2016, p. 37).

Dedicado a una perspectiva regional, también es necesario destacar el breve estudio de la UMC (2016), con datos de la UMC-EM 2013. Este estudio se concentra en la elaboración de modelos específicos del rendimiento diferenciados según las tres regiones geográficas: costa, sierra y selva. Estas regiones incorporan las 26 regiones administrativas usuales. Los modelos incluyen

3. Este informe está disponible en $<\mathrm{http}$ //umc.minedu.gob.pe/evaluaciones-muestrales/ $>$. 
factores asociados contextuales del alumno y su familia, el docente y el director de la escuela. Además de presentar las diferencias entre las tres regiones naturales del país, el mencionado estudio destaca la incidencia de factores como el género del alumno, la motivación y expectativas del alumno y padres, el índice socioeconómico (ISE) del alumno y de la escuela, así como la formación y satisfacción laboral del docente.

\section{Marco metodológico}

\section{La base de datos y muestra}

La base de datos disponible públicamente proviene de la UMC-EM 2013, encuesta muestral del Ministerio de Educación del Perú, aplicada a los alumnos del último grado de educación primaria (sexto grado) en las escuelas representativas de todo el país para medir las competencias de Lectura, Matemáticas y Ciudadanía ${ }^{4}$. La aplicación de cuestionarios de contexto permitió sondear aspectos tales como las características de los alumnos, su familia, los docentes y los directores. La muestra pública disponible fue de 66,584 observaciones. A fin de uniformizar las respuestas en las pruebas, en este trabajo, se ha seleccionado los datos de aquellos alumnos que han respondido a las tres pruebas. De este modo, la muestra final resulta en 62,849 observaciones correspondientes a 3017 escuelas.

Como es propio de las encuestas, la ausencia de información en los cuestionarios puede generar sesgos que afectan la inferencia estadística. Estos "valores perdidos" pueden ser producto de preguntas no aplicadas, errores de medición y de muestreo, confiabilidad de preguntas y respuestas, registro defectuoso de respuestas, entre otras razones. Aunque presentes en la base de datos, en este trabajo, no se ha considerado usar metodologías particulares para paliar la ausencia de información de estos valores perdidos.

El informe técnico (UMC, 2014) detalla la muestra probabilística compleja, bietápica por conglomerados y estratificada que sustenta la encuesta muestral. Una estratificación por tamaño de conglomerado incorpora de manera independiente cada Dirección Regional de Educación. Además, se consideran como estratos implícitos la gestión, el área de localización y las características monodocente/polidocente. En este diseño, un conglomerado es una institución educativa, la escuela. La selección de las escuelas en dos etapas - sistemática y, luego, aleatoria simple - permite niveles de inferencia con un margen de error aceptable. A partir del análisis exhaustivo de las respuestas a los ítems, de acuerdo con sus diversos grados de dificultad, evidencias de confiabilidad y validez, la UMC-EM 2013 construyó una escala numérica generada por el modelo psicométrico de Rasch. Este modelo facilita la interpretación de los

4. También, se aplicó una prueba especial de escritura con un protocolo diferente a las demás pruebas de competencias. Los resultados figuran en otra base de datos. 
resultados, mediante una escala de habilidad cuyo valor promedio convencionalmente ha sido fijado en 500 puntos y con una desviación estándar en 100 puntos.

Cabe mencionar la disponibilidad de un índice socioeconómico del alumno. Este índice oficial sintético - también, denominado ISE - es de gran utilidad para tratar de estimar las características del alumnado que pertenece a la misma situación social y constituye, en este trabajo, el instrumento principal que sirve de viga maestra para el análisis. El ISE utiliza información proveniente de los cuestionarios de factores asociados, y está constituido por un índice de vivienda y acceso a servicios, un índice de recursos educativos en el hogar y el nivel educativo de los padres. El índice es jerárquico, en la medida que sus valores más altos corresponderían a mejores condiciones socioeconómicas. Para uniformizar la diversidad de los elementos que entran en su construcción, el índice ha sido estandarizado a un valor promedio de 0 y una desviación estándar de 1; de esta manera, ha conservado métrica de variable continua. Estos ítems fueron sometidos a un análisis estadístico de componentes principales para datos categóricos y una amplia presentación se encuentra en el Reporte Técnico (UMC, 2015).

Su utilidad se puede extender hacia el centro educativo, pues se puede elaborar un ISE-escuela, sobre la base del promedio del puntaje ponderado del índice socioeconómico de los alumnos pertenecientes a cada escuela. Los valores de estos índices correspondientes a cada región figuran en el Anexo 1.

\section{Metodología}

Como es usual, para respetar el carácter muestral de la encuesta, se considera la variable peso muestral, que es el peso probabilístico del alumno en la muestra dentro de la población. Luego, se incorpora la variable unidad primaria de muestreo, que corresponde a la escuela y el estrato muestral que particiona a la población estudiantil en diferentes grupos. Estos parámetros permiten calcular la linealización de los estimados de la varianza. Esta información está disponible en la base pública de datos.

La selección de las variables está basada en la evidencia examinada en la sección de revisión de la literatura. Por eso, como variables dependientes, se utilizan los puntajes de los alumnos en cada una de las pruebas y, como covariables asociadas, se ha seleccionado el ISE-alumno para analizar los efectos de composición social. La mayoría de los análisis utilizan modelos estadísticos lineales generalizados en su vertiente de regresión ordinaria lineal y regresión multinivel.

El modelo básico es el siguiente:

$$
\begin{aligned}
& \mathrm{Y}_{\mathrm{i}}=\beta_{\mathrm{o}}+\beta_{1} \mathrm{X}_{\mathrm{i} 1}+\beta_{2} \mathrm{X}_{\mathrm{i} 2}+\ldots \beta_{\mathrm{p}} \mathrm{X}_{\mathrm{ip}}+\varepsilon_{\mathrm{i}} \\
& \text { En esta fórmula, cada observación }=1, \ldots, \mathrm{n} .
\end{aligned}
$$


La fórmula considera las $n$ observaciones de la variable dependiente y las $p$ variables independientes. Entonces, $\mathrm{Y}_{\mathrm{i}}$ es la i observación de la variable dependiente y $\mathrm{X}_{\mathrm{ij}}$ es la i observación de la $\mathrm{j}$ variable independiente, $\mathrm{j}=1,2, \ldots, \mathrm{p}$. Los valores beta $\beta_{\mathrm{i}}$ representan los parámetros a estimar y $\varepsilon_{i}$ es el error normal de i.

Para respetar la estructura jerárquica de los datos de la encuestas -es decir, de alumnos dentro de la escuela-, en el caso de estimación simultánea de los dos índices socioeconómicos dentro de una ecuación lineal, se ha aplicado la regresión multinivel (Snijders y Bosker, 2012), que considera que los alumnos se agrupan (están anidados) en escuelas. El modelo jerárquico lineal o mixto - denominado HLM por sus siglas en inglés- permite identificar la proporción de la varianza del rendimiento académico que ocurre intraescuelas e interescuelas (correlación intraclase o ICC), como se presentará más adelante.

La ecuación es la siguiente: $\mathrm{Yij}=\gamma 00+\gamma 10\left(\mathrm{X}_{\mathrm{ij}}\right)+[\mathrm{u} 0 \mathrm{j}+\mathrm{rij}]$

En dicha ecuación, el término u0j indica el efecto aleatorio a nivel 2, lo cual representa la variación en el promedio del puntaje respectivo interescuelas. El término $r_{i j}$ es el efecto aleatorio a nivel 1, que refleja la variación en el promedio del puntaje respectivo intraescuelas.

La varianza en los rendimientos académicos que ocurre entre las escuelas y la varianza de los residuos que ocurre al interior de las escuelas son conocidas como la varianza interescuela e intraescuela, respectivamente. La suma de ambas es la varianza total de los logros. La relación entre ambas varianzas se mide por el coeficiente "rho" $(\rho)$, llamado también "coeficiente de correlación intraclase" (ICC). Dicho coeficiente se formula como la ratio de la varianza entre escuelas con respecto a la varianza total y se expresa en la siguiente relación:

$$
\rho=\frac{\tau_{00}}{\tau_{00}+\sigma^{2}}
$$

Los puntajes alcanzados en las pruebas han sido ordenados y clasificados según cuatro niveles de rendimiento. A partir de un análisis exhaustivo de los ítems, de acuerdo con sus diversos grados de dificultad, se ha construido cuatro niveles de logro. Estos niveles deben reflejar la capacidad del alumno con respecto a la competencia o a la habilidad que está siendo medida, y determinar si los desempeños observados pueden ser considerados satisfactorios según las competencias esperadas al finalizar el quinto ciclo de la Educación Básica Regular.

Para las comparaciones del rendimiento, el nivel considerado como "satisfactorio" sirve como base de referencia en este estudio y es el nivel en el cual el alumno logró los aprendizajes esperados para el grado que cursa. En el nivel "en proceso", el alumno se encuentra en vías de alcanzar los logros esperados, 
que están en una fase inicial del desarrollo de sus aprendizajes y que responden solo las preguntas más sencillas de la prueba. Finalmente, en el nivel "por debajo del inicio", se ubican los alumnos que no logran alcanzar los aprendizajes esperados, que se encuentran en una fase muy inicial de sus aprendizajes y que evidencian dificultades para responder incluso a las preguntas más fáciles de la prueba.

\section{Resultados}

Los puntajes alineados a un promedio de 500 puntos y una desviación estándar de 100 se completan con el establecimiento de los niveles de rendimiento. Para fines de comparación, se opta como línea de base la categoría "satisfactorio" para todas las competencias. La siguiente tabla permite constatar que el logro satisfactorio en cada una de las competencias es más bien modesto: $16 \%$ en matemáticas; 21.3 en lectura y $30.4 \%$ en ciudadanía ${ }^{5}$. Los promedios de los puntajes expresados en valores de escala continua reflejan esta situación. Desde luego, es posible encontrar a un alumno con alto/bajo rendimiento en una competencia y con alto/bajo rendimiento en la otra u otras dos competencias; esta posibilidad da lugar a una serie de combinaciones.

Tabla 1. Puntajes promedio y porcentaje del nivel de rendimiento en Matemáticas, Lectura y Ciudadanía

\begin{tabular}{lcrr}
\hline & Matemáticas & Lectura & Ciudadanía \\
\hline Promedio nacional & 501.1 & 500.4 & 501.0 \\
& $(1.712)$ & $(1.582)$ & $(1.550)$ \\
Promedio & & & \\
Satisfactorio & 657.6 & 633.8 & 615.4 \\
& $(1.124)$ & $(1.029)$ & $(0.934)$ \\
No satisfactorio & 470.6 & 464.1 & 450.1 \\
& $(0.870)$ & $(0.924)$ & $(0.763)$ \\
\% Satisfactorio & 16.3 & 21.4 & 30.9 \\
& $(0.006)$ & $(0.006)$ & $(0.006)$ \\
\% No satisfactorio & 84.7 & 71.6 & 69.2 \\
& $(0.006)$ & $(0.006)$ & $(0.006)$ \\
\hline
\end{tabular}

N.B.: Errores estándar entre paréntesis

Todos los valores son estadísticamente significativos a $\mathrm{p}<0.001$.

Fuente: Elaboración propia, a partir de la base de datos UMC-EM 2013

5. Estos porcentajes coinciden con los de la UMC-EM 2013 (UMC, 2016): Tabla 3.3 (Lectura), Tabla 3.15 (Matemáticas) y Tabla 3.23 (Ciudadanía). 
Teniendo en cuenta los puntajes obtenidos simultáneamente en las tres pruebas, se puede afirmar que solamente $9.2 \%$ de alumnos tienen resultados satisfactorios en todas las pruebas, el $62.2 \%$ - un porcentaje importante de ellos- obtiene resultados no satisfactorios en todas las pruebas, y el $28.6 \%$ corresponde al saldo de las combinaciones posible de competencias y niveles. Estas cifras reflejan ciertamente un panorama poco alentador. Frente a dicho panorama, conviene incorporar inmediatamente al análisis — bajo diferentes formas- el impacto que tiene el índice socioeconómico.

\section{Relaciones entre el índice socioeconómico y el rendimiento académico}

El análisis de regresión multinivel permite estimar la importancia de las relaciones entre el índice y los resultados en las tres competencias respetando el carácter anidado de los datos. A partir de este análisis, por un lado, es posible apreciar la varianza de los puntajes entre alumnos y escuela cuando no interviene el índice en la determinación de los resultados, lo que es denominado "modelo nulo". Por otro lado, facilita observar la varianza cuando hay influencia de este índice, lo que es conocido como "modelo mixto".

Tabla 2. Modelo nulo y mixto de relaciones entre los puntajes de Matemáticas, Lectura y Ciudadanía, y el índice socioeconómico del alumno (ISE)

\begin{tabular}{|lrrr|}
\hline & Matemáticas & Lectura & Ciudadanía \\
\hline Coeficiente ISE alumno & 21.7 & 24.1 & 22.9 \\
& $(0.802)$ & $(0.782)$ & $(0.772)$ \\
\hline Constante & 486.2 & 483.5 & 486.3 \\
& $(1.100)$ & $(1.007)$ & $(1.020)$ \\
\hline Modelo nulo - ICC (\%) & 47.7 & 51.4 & 44.8 \\
& $(0.006)$ & $(0.006)$ & $(0.00)$ \\
Modelo mixto - ICC (\%) & 38.9 & 40.1 & 34.4 \\
& $(0.006)$ & $(0.007)$ & $(0.006)$ \\
\hline
\end{tabular}

N.B: Errores estándar entre paréntesis

Todos los valores son estadísticamente significativos a $\mathrm{p}<0.001$.

Fuente: Elaboración propia, a partir de la base de datos UMC-EM2013.

El coeficiente de la recta de regresión, denominado "pendiente" o "gradiente" (Willms, 2006), permite apreciar el grado de impacto que ejerce este índice sobre los logros. Así, ceteris paribus ${ }^{6}$, el aumento de una unidad en el índice genera un incremento de 21.7 puntos en el rendimiento promedio de Matemáticas, 24.1 en Lectura y 22.9 en Ciudadanía. Estas relaciones pueden visualizarse con la representación gráfica de las rectas de mejor ajuste de las

6. En el contexto modelístico, esta expresión latina significa «todo lo demás constante». 
regresiones. Como es evidente, a medida que aumenta el valor del índice, mayores son puntajes de rendimientos continuos y positivos en todas las competencias, sin excepción.

Gráfico 1.

Relación entre los puntajes promedios de Lectura, Matemáticas y Ciudadanía, y el índice socioeconómico del alumno

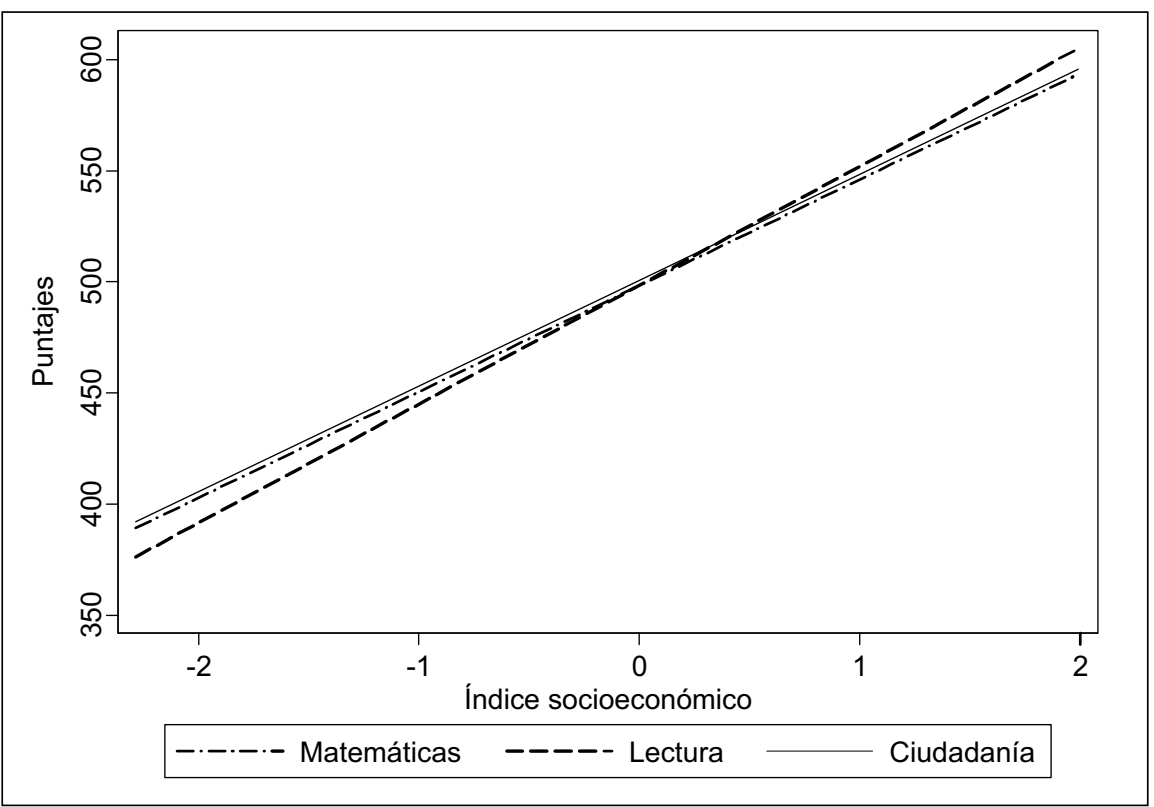

Todos los valores son estadísticamente significativos a $\mathrm{p}<0.001$.

Fuente: Elaboración propia, a partir de la base de datos UMC-EM 2013.

Las ecuaciones que estructuran los parámetros del Gráfico 1 se formulan de la siguiente manera:

$\begin{array}{ll}\text { Matemáticas: } & 21.7^{*} \text { índice }+486.2 \\ \text { Lectura: } & 24.1^{*} \text { índice }+483.5 \\ \text { Ciudadanía: } & 22.9^{*} \text { índice }+486.3\end{array}$

Por su parte, el valor del ICC (coeficiente de correlación intraclase) permite apreciar la variabilidad del rendimiento según los modelos. Así, el modelo nulo informa que, entre $44.8 \%$ y $51.4 \%$ de los rendimientos, se explica por la influencia de factores no observados que operan en el interior de las escuelas. La otra mitad se explica por las diferencias de factores no observados que pertenecen al alumno. Sin embargo, cuando interviene la condición social 
del alumno (modelo mixto), la influencia disminuye a un rango entre 34.4\% y $40.1 \%$. Dicha influencia es siempre importante para explicar la varianza que ocurre en las escuelas, tratándose de un único factor del modelo.

\section{El perfil socioeconómico de alumnos y escuelas}

Por su carácter de variable continua, el índice socioeconómico del alumno (ISE-alumno) permite dividir su distribución por cuartiles (4 proporciones iguales de $25 \%$ ) y sobre esta base, se puede clasificar convencionalmente la posición social de los alumnos. Los alumnos desfavorecidos son aquellos que se encuentran en el primer cuartil del índice socioeconómico; los alumnos promedios, aquellos que pertenecen al segundo y tercer cuartil; y los alumnos favorecidos, aquellos que se ubican en el primer cuartil.

De igual manera y convencionalmente, se procede con el índice socioeconómico de la escuela (ISE-escuela). Este índice intenta representar sintética y estadísticamente el perfil social del centro escolar. El primer cuartil agrupa a las escuelas desfavorecidas; en el segundo y el tercer cuartil, están categorizadas las escuelas mixtas; y, en el cuartil superior, se encuentran las escuelas favorecidas. De esta manera, se obtiene una distribución cruzada de nueve categorías que figuran en la siguiente tabla. El promedio nacional del ISE-escuela es de 0.0182 (e.e=0.018) y el del ISE alumno de 0.0207 (e.e=0.018). La tabla 3 presenta, a doble entrada, la distribución porcentual de las categorías de alumnos y escuelas.

Tabla 3. Porcentajes por escuelas y alumnos, según competencias y composición socioeconómica

\begin{tabular}{lrrr}
\hline & \multicolumn{1}{c}{$\begin{array}{c}\text { Alumnos } \\
\text { desfavorecidos }\end{array}$} & \multicolumn{1}{c}{$\begin{array}{c}\text { Alumnos } \\
\text { promedio }\end{array}$} & $\begin{array}{c}\text { Alumnos } \\
\text { favorecidos }\end{array}$ \\
\hline Escuelas desfavorecidas & 76.6 & 11.4 & 0.2 \\
Escuelas mixtas & $(0.835)$ & $(0.507)$ & $(0.046)$ \\
Escuelas favorecidas & 23.2 & 72.9 & 29.7 \\
\% Total nacional & $(0.836)$ & $(1.446)$ & $(2.008)$ \\
\hline
\end{tabular}

N.B: Errores estándar entre paréntesis

Todos los valores son estadísticamente significativos a $\mathrm{p}<0.001$.

Fuente: Elaboración propia, a partir de la base de datos UMC-EM 2013

La distribución de alumnos y escuelas presenta un patrón evidente. Las tres cuartas partes de alumnos socialmente desfavorecidos (76,6\%) asisten igual140 I mente a escuelas socialmente desfavorecidas. En el extremo opuesto, 70,1\% 
de alumnos socialmente favorecidos frecuentan escuelas también socialmente favorecidas. Este resultado puede ser un considerado un indicador bruto de la reproducción social en el acceso a las escuelas. También, se debe señalar la presencia casi nula $(0.2 \%)$ de alumnos desfavorecidos en escuelas favorecidas, así como es igualmente nula la presencia de alumnos favorecidos que asisten a escuelas desfavorecidas $(0,2 \%)$. Para completar el perfil de alumnos y escuelas, la siguiente tabla expresa esta distribución en valores de puntajes.

Tabla 4. Puntajes promedio por escuelas y alumnos, según competencias y composición socioeconómica

\begin{tabular}{lrcrrrrrrr}
\hline & \multicolumn{3}{c}{ Matemáticas } & \multicolumn{3}{c}{ Lectura } & \multicolumn{3}{c}{ Ciudadanía } \\
\hline Alumnos & \multicolumn{1}{c}{$\mathrm{D}$} & $\mathrm{P}$ & \multicolumn{1}{c}{$\mathrm{F}$} & $\mathrm{D}$ & \multicolumn{1}{c}{$\mathrm{P}$} & \multicolumn{1}{c}{$\mathrm{F}$} & \multicolumn{1}{c}{$\mathrm{D}$} & \multicolumn{1}{c}{$\mathrm{P}$} & \multicolumn{1}{c}{$\mathrm{F}$} \\
\hline Escuelas D. & 424.8 & 467.6 & 485.3 & 409.0 & 442.0 & 497. & 417.5 & 449.7 & 500.5 \\
& $(1.986)$ & $(2.05)$ & $(16.72)$ & $(1.768)$ & $(2.342)$ & $(15.5)$ & $(1.726)$ & $(2.458)$ & $(17.09)$ \\
Escuelas M. & 447.6 & 502.6 & 538.1 & 463.1 & 503.8 & 550.9 & 471.1 & 505.8 & 548.1 \\
& $(2.394)$ & $(1.82)$ & $(3.879)$ & $(1.885)$ & $(1.521)$ & $(2.543)$ & $(1.37)$ & $(1.533)$ & $(2.432)$ \\
Escuelas F. & 500.7 & 543.2 & 578.5 & 500.1 & 547.9 & 585.2 & 505.1 & 541.1 & 573.9 \\
& $(18.73)$ & $(2.71)$ & $(3.609)$ & $(16.33)$ & $(2.672)$ & $(2.905)$ & $(15.47)$ & $(4.383)$ & $(2.948)$ \\
\hline
\end{tabular}

N.B: Errores estándar entre paréntesis

Todos los valores son estadísticamente significativos a $\mathrm{p}<0.001$.

Fuente: Elaboración propia, a partir de la base de datos UMC-EM 2013

Como es de esperar, los alumnos socialmente favorecidos que asisten a las escuelas socialmente favorecidas destacan sistemáticamente por sus mejores resultados en todas las competencias. Al contrario, los alumnos desfavorecidos que atienden a escuelas socialmente desfavorecidas obtienen sistemáticamente peores resultados. Como señala Willms (2006), este efecto de composición socioeconómica produce un doble riesgo para los alumnos desfavorecidos, pues estos son desfavorecidos social y académicamente. Así, por ejemplo, un alumno desfavorecido que asiste a una escuela desfavorecida alcanza 424.8 puntos en Matemáticas. Por su parte, el alumno favorecido que asiste a una escuela favorecida alcanza 578.5 puntos en la misma disciplina. La diferencia representa, entonces, 153.7 puntos en Matemáticas. Esta diferencia asciende a 176.2 en Lectura y 156.4 puntos en Ciudadanía. Al respecto, cabría notar la situación particular de la competencia de lectura, en que la diferencia es mayor.

Aunque se ha constatado la escasa presencia de alumnos socialmente desfavorecidos en las escuelas favorecidas y a pesar de esta paradójica posibilidad, ellos se benefician con 75.9 puntos más en Matemáticas, 91.1 más en Lectura y 87.6 puntos en Ciudadanía. El siguiente gráfico facilita la lectura de las nueve rectas posibles. 
Gráfico 2.

Puntajes promedios según competencias y condición socioeconómica de escuelas y alumnos

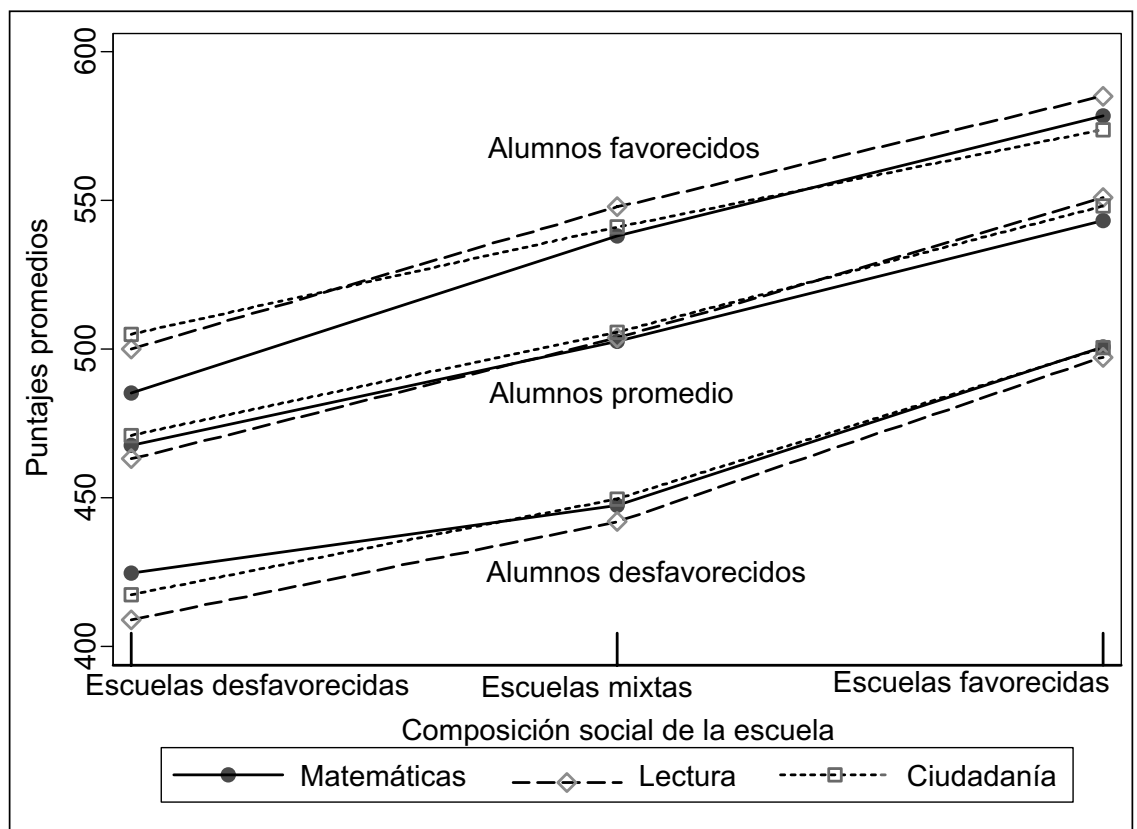

N.B:Todos los valores son estadísticamente significativos a $\mathrm{p}<0.001$.

Fuente: Elaboración propia, a partir de la base de datos UMC-EM 2013

Esta constatación refuerza lo planteado en el informe oficial, según el cual "las diferencias en el rendimiento de los estudiantes peruanos se asocian, sobre todo, a la composición socioeconómica del grupo evaluado que intensifica el efecto del índice socioeconómico individual. Al parecer esta situación se ha mantenido en la última década" (UMC, 2016, p. 141). Establecida esta relación a nivel de un agregado como es la institución escolar, inmediatamente, surge la pregunta acerca de cuán importante son el impacto y la fuerza de esta relación, es decir, si los rendimientos observados son atribuibles a las propias escuelas o si estos provienen de los propios alumnos.

\section{La distribución del rendimiento por regiones}

Desde la perspectiva de la región, vale citar los propósitos del Consejo Nacional de Educación (CNE, 2007, p. 96), que aboga por una gestión eficaz, ética, descentralizada y con participación ciudadana. El cuarto objetivo estratégico planteado por el CNE propugna una gestión que logra buenos resultados, y es

142 I financiada con equidad y eficiencia. Por eso, es necesario mostrar la dimensión de estas desigualdades de rendimiento entre regiones. 
En el siguiente gráfico, se puede distinguir, en primer lugar, que - de las 26 regiones existentes en el país - hay 10 regiones que se sitúan por encima del promedio nacional fijado a 500 puntos y 16 regiones por debajo de este. En este caso, Tacna destaca visiblemente como la región de mayor rendimiento de alumnos en las tres competencias. En el extremo, Loreto es el más alejado del promedio nacional en Matemáticas, que acompaña en la misma posición a Huancavelica, pero esta vez en Lectura y Ciudadanía. En segundo lugar, se constata que el solape de puntajes entre las tres competencias es común, pero hay regiones (Ucayali, Loreto, Huancavelica y Madre de Dios) cuyos logros son bastante dispersos entre las disciplinas. Este rango de dispersión es de tal envergadura que las distancias ascienden a más de 150 puntos de diferencia entre Loreto y Tacna en Matemáticas, y entre Moquegua y Huancavelica en lo que respecta a Lectura.

\section{Gráfico 3.}

Distribución de puntajes promedios en Matemáticas, Lectura y Ciudadanía, según regiones

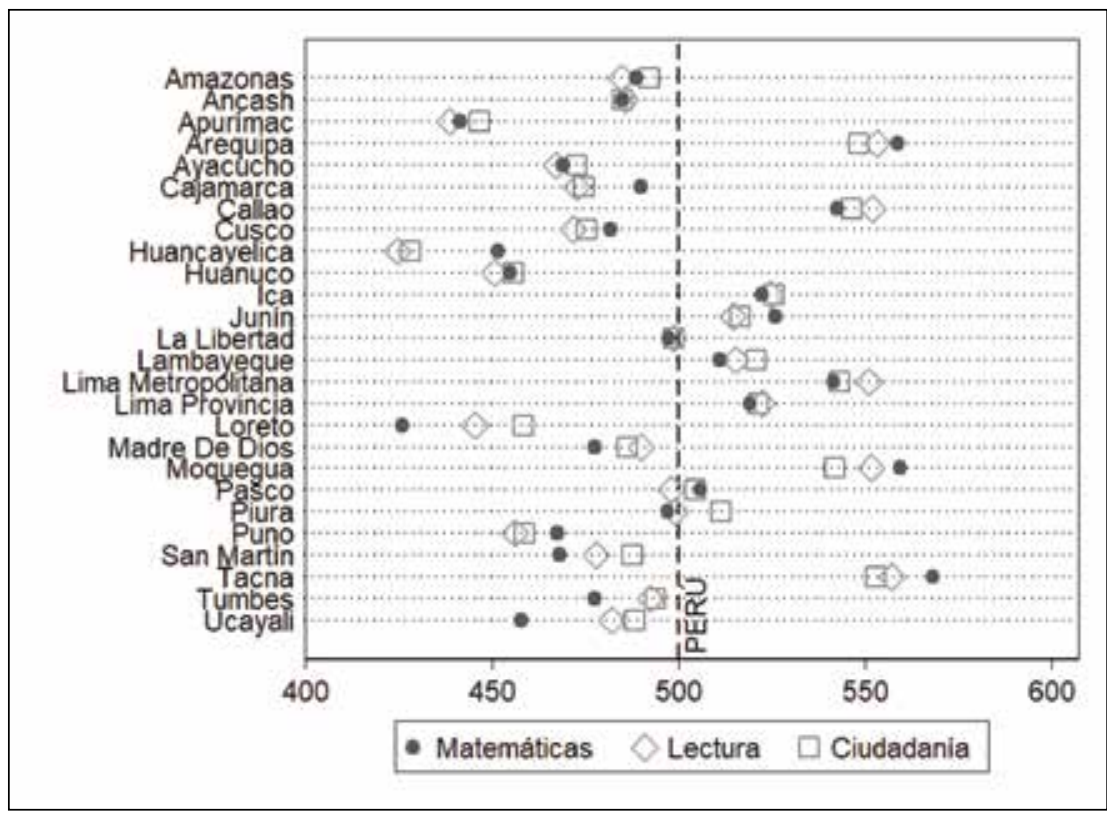

N.B: En el Anexo 1, se presenta los valores de los puntajes y errores estándares en las tres competencias.

Todos los valores son estadísticamente significativos a $\mathrm{p}<0.001$.

Fuente: Elaboración propia, a partir de la base de datos UMC-EM 2013 
Esta severa doble desigualdad, interregional e intrarregional, de los logros regionales de aprendizaje advierte la envergadura de los desafíos pendientes que han de enfrentar las políticas educativas con espíritu de justicia y equidad regional.

\section{La composición social de las escuelas por regiones}

En el acápite anterior, se ha presentado la fuerte dispersión de los rendimientos entre las regiones. Frente a ello, es necesario prestar interés a la distribución de la composición social de las escuelas según estas regiones con el propósito de explorar eventuales asociaciones explicativas a los rendimientos desiguales.

El siguiente gráfico muestra la importante diversidad de la composición social, con un promedio nacional de $21 \%$ de escuelas socialmente desfavorecidas. En este, se observa que quince regiones superan este promedio. La mitad de las regiones acogen poblaciones desfavorecidas. Sin embargo, se debe alertar que Huancavelica se compone de $70 \%$ de estas escuelas, seguida de cerca por Cajamarca, con $66 \%$.

\section{Gráfico 4.}

Perú: Distribución porcentual de la composición social de las escuelas, por regiones

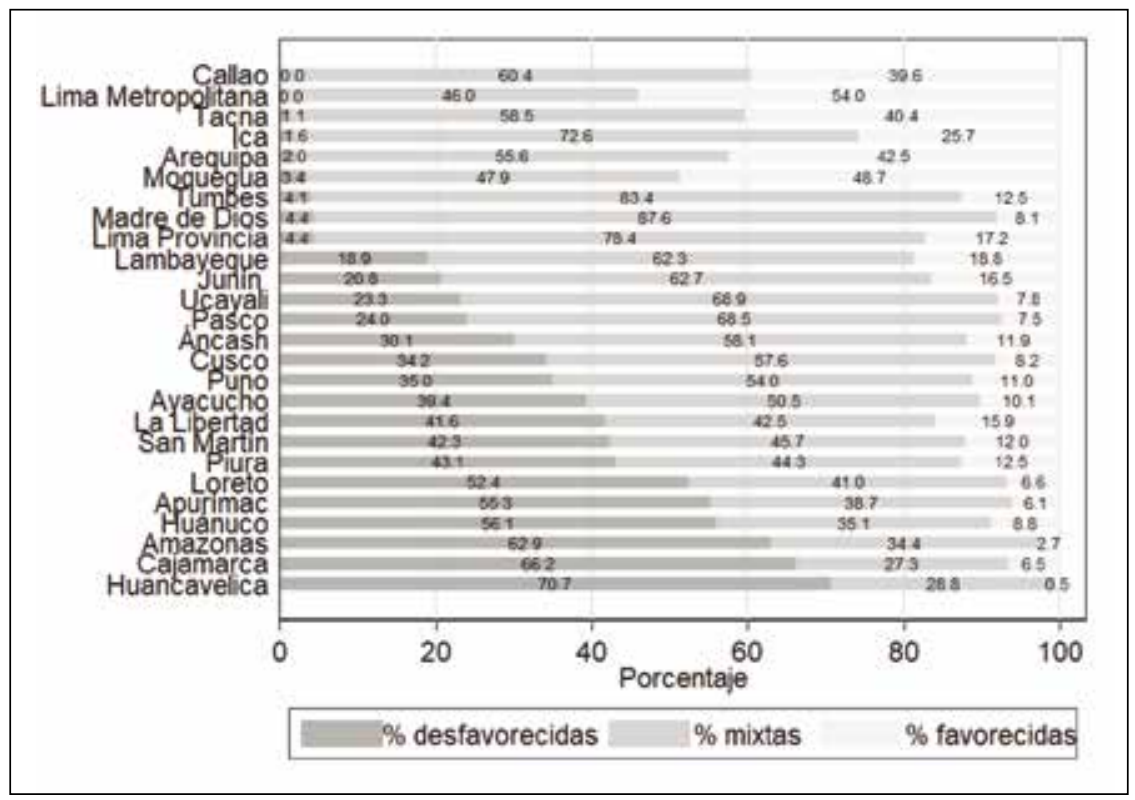

N.B: Todos los valores son estadísticamente significativos a $\mathrm{p}<0.001$.

Fuente: Elaboración propia, a partir de la base de datos UMC-EM 2013 
Llama la atención que, según el criterio ISE empleado en este caso, el Callao y Lima Metropolitana no poseerían escuelas socialmente desfavorecidas y, también, existiría regiones que poseen muy bajos porcentajes de estas escuelas (de hasta menos del 5\%) ${ }^{7}$. Si bien estos resultados pueden despertar dudas sobre la pertinencia y la representatividad del índice utilizado, a su vez, invitan a mejorar aquellos índices - nivel socioeconómico, ocupación y educación de los padres, etc. - que están en vigencia y, tal vez, a construir otros índices sociales que abarquen nuevos criterios de cobertura para estimar eficazmente la situación social que rodea los aprendizajes.

\section{Impacto del ISE-alumno según el rendimiento de las regiones}

Hay consenso en que un sistema educativo será tanto más equitativo cuanto el incremento del índice social genere menor variación en los resultados, puesto que reproduciría en menor medida el efecto social; de este modo, garantizaría una mayor igualdad de oportunidades. En este trabajo, se aborda una medida clave: el impacto (gradiente) de este índice en las distintas disciplinas y regiones. Los valores betas de las gradientes permiten tener un enfoque analítico de las desigualdades académicas relacionadas con la condición social. Los resultados ayudan a entender cómo se configuran estas desigualdades entre las escuelas y entre alumnos (Caro y Lenkeit, 2012; Willms, 2006). Como es usual, una gradiente (pendiente) inclinada indica un mayor impacto del índice sobre el logro académico y expresa mayor dependencia social del alumno que si la pendiente fuera menos empinada. El valor de una gradiente más plana indica comparativamente mayor equidad.

Cabe recordar que el impacto del coeficiente $\beta$ a nivel nacional alcanza 21 puntos en Matemáticas, 24 puntos en Lectura y 22 en Ciudadanía (Tabla 2). Estos coeficientes representan los beneficios generados en los puntajes promedios del alumno gracias al impacto de la condición social. Tratándose de valores a nivel del país, es de esperar una variabilidad por cada región semejante. Sin embargo, el siguiente gráfico presenta un panorama sorprendente (los datos figuran en el Anexo 2). Los coeficientes tienen en cuenta el carácter multinivel/mixto de la estructura de los datos.

7. Estas, precisamente, obtienen buenos resultados promedios en todas las competencias. 


\section{Gráfico 5.}

Impacto (coeficiente $\beta$ ) de la relación entre índice socioeconómico de los alumnos y el rendimiento en Matemáticas, Lectura y Ciudadanía, según regiones

\begin{tabular}{|c|c|c|c|c|c|}
\hline \multicolumn{2}{|c|}{ MATEMÁTICAS } & & LECTURA & \multicolumn{2}{|r|}{ CIUDADANIA } \\
\hline Pasco & $=370$ & Cequmarca & 307 & Ancash & aras 7 \\
\hline Ancash & 344 & Äncash & $=376$ & Pasco & $=37.7$ \\
\hline Puno & ast 3 & Paseo & 390 & Cajamarca & -2363 \\
\hline Cajamarca & an 206 & Puno & 134.6 & San Martín & 328 \\
\hline Arequipa & $=29.4$ & Lima Provincias & $=39.5$ & Cusco & 326 \\
\hline Lima Provincias & 28.2 & Cusco & 334 & Lima Provincias & 032.2 \\
\hline Moguegua & 250 & Sen Martin & $=328$ & Puno & 3316 \\
\hline Madre de Dios & 3268 & Ayacucho & 326 & Amazonas & 1312 \\
\hline Lima Metropoltana & 3267 & Junín & $=2+0$ & La Libertad & 257 \\
\hline Cusco & 3266 & Anazonas & 391.5 & Apurimac & 9287 \\
\hline Junin & 2253 & Madre de Dios & 310 & Junin & 26.3 \\
\hline Ayocucho & 251 & Apurimac & 30.8 & Piura & 282 \\
\hline Huánuco & 250 & La Libertad & 2303 & Huảnuco & 27.1 \\
\hline San Martín & 246 & Piera & 3902 & Lima Metropolsana & 2271 \\
\hline Amagonas & 240 & Iea & 3002 & Ayacueho & 36287 \\
\hline Ica & $=238$ & Lima Metropolitana & 286 & Lambayeque & 257 \\
\hline La Libertad & 23.2 & Lambayeque & $=28.1$ & Ica & 25.7 \\
\hline Lambayeque & $\mathbf{3} 23.2$ & Moquegua & $\ln 269$ & Madre de Dios & 25.4 \\
\hline Piura & 3.22 .0 & Hudnuco & $m 258$ & Tumbes & 224.8 \\
\hline Apurimac & 1226 & Calao & 256 & Arequipa & 247 \\
\hline Calao & 222.3 & Arequipa & 3254 & Moquegua & 22.9 \\
\hline Tacna & 220 & Ueayal & 0251 & Ucayali & 228 \\
\hline Tumbes & 2210 & Tumbes & $\cos 20$ & Callaso & 21.4 \\
\hline Ucayali & $=210$ & Huancavelica & 3232 & Huancavelica & 21.4 \\
\hline Loreto & 3166 & Tacna & 215 & Tacna & 200 \\
\hline Huancanelica & 148 & Loreto & 200 & Loreto & 185 \\
\hline & $\begin{array}{l}10.2030,40 \\
\text { Pendiente }\end{array}$ & & 0 to 203040 & & 0 Pendiente \\
\hline
\end{tabular}

N.B: En el Anexo 2, se presenta los valores de los coeficientes y errores estándar en las tres competencias.

Todos los valores son estadísticamente significativos a $\mathrm{p}<0.001$.

Fuente: Elaboración propia, a partir de la base de datos UMC-EM 2013

Los coeficientes de regresión ordenados por sus valores descendentes reflejan un impacto mucho mayor para cada región que el índice promedio nacional, en el que casi todas las regiones acusan una influencia significativa de la condición social de los alumnos. Los contrastes son importantes entre regiones como Pasco, Cajamarca y Áncash, que se benefician en aproximadamente 40 puntos en rendimiento por cada unidad de aumento de sus respectivos índices ISE, frente al promedio nacional, que otorga solo un beneficio de 20 puntos en general, sin distinción de región. En el otro extremo, existiría menor influencia de la posición social en Loreto y Huancavelica, que obtienen beneficios menores. Sin embargo, se debe notar que este menor beneficio ocurre también en Tacna, que es una región con alto ISE.

Existen regiones con mayor equidad, es decir, que son más igualitarias socialmente por el bajo impacto del índice social, pero obtienen bajos resultados. Es sabido que conseguir una mayor equidad social favorece, mas no garantiza, mejores logros y es posible que se pueda alcanzar un alto rendimiento conservando igualmente una alta equidad. Puede ocurrir, asimismo, que se brin146 I den condiciones de aprendizaje socialmente igualitarias, aunque sin garantizar 
altos logros. Los coeficientes presentados pretenden identificar este impacto social.

\section{Las diferenciaciones de la gestión pública y privada}

En un trabajo sobre desigualdad social en las regiones parece pertinente, aun sin profundizar, abordar el tema de la gestión escolar. Cabe recordar que, en UMC-EM 2013, participaron 77,1\% alumnos del sector estatal y $22,9 \%$ del no estatal. En todas las competencias medidas en dicha encuesta, el promedio de rendimiento en la gestión no estatal es sistemáticamente mayor: Matemáticas (556 versus 484), Lectura (565 versus 480 ) y Ciudadanía (551 versus 485). Cuando interviene el índice ISE-alumno en la relación gestión-rendimiento, los coeficientes de la regresión multinivel indican la fuerte influencia de la variable tipo de gestión. En efecto, la siguiente tabla registra los beneficios de la condición social del alumno, que asciende a más de 20 puntos en cada competencia. Sin embargo, tomando como referencia los alumnos de escuelas estatales, se evidencia que estos beneficios son mucho más sensibles para los alumnos de las escuelas no estatales, quienes obtienen beneficios tan importantes que van desde 46.9 en Ciudadanía hasta 64.3 en Lectura. De esta manera, se confirma la doble ventaja social: los alumnos tienen mejores rendimientos gracias a la ventaja de su origen social y a su ventaja por asistir a escuelas de gestión no estatal.

Tabla 5. Impacto del índice socioeconómico según competencia por gestión no estatal y estatal, e índice intraclase (ICC)

\begin{tabular}{lrrrrrr}
\hline & \multicolumn{2}{c}{ Matemáticas } & \multicolumn{2}{c}{ Lectura } & \multicolumn{2}{c}{ Ciudadanía } \\
\hline & Coeficiente & e.e & Coeficiente & e.e & Coeficiente & e.e \\
\hline Índice ISE-alumno & 21.4 & 0.807 & 23.8 & 0.787 & 22.6 & 0.783 \\
$\begin{array}{l}\text { Gestión } \\
\text { No estatal } \\
\text { (base = Estatal) }\end{array}$ & 53.1 & 2.56 & 64.3 & 2.307 & 46.9 & 2.475 \\
Constante & & & & & & \\
\hline ICC (\%) & 477.1 & 1.235 & 472.4 & 1.197 & 478.2 & 1.161 \\
\hline
\end{tabular}

N.B.: En el Anexo 3, se presenta los valores de los coeficientes y errores estándar en las tres competencias.

Todos los valores son estadísticamente significativos a $\mathrm{p}<0.001$.

Fuente: Elaboración propia, a partir de la base de datos UMC-EM 2013

En cuanto a la variabilidad al interior de las escuelas, la comparación de los valores del ICC con aquellos generales de la Tabla 2 permite apreciar una ligera disminución cuando se incorpora la variable gestión. En esa medida, la variabilidad en los rendimientos por impacto del índice socioeconómico 
sigue siendo igualmente importante cuando se distingue las escuelas por su condición de no estatales.

Es necesario anotar que este cuadro general sobre el tema de la gestión parece discutible, pues la literatura académica ha detectado - particularmente, en la capital del país - la existencia de escuelas privadas con logros iguales o menores que aquellos de las escuelas públicas en distritos de nivel socioeconómico modesto (Balarin, 2015; Cuenca, 2013). En ciertos distritos, cuando los efectos del nivel socioeconómico individual y de la composición social de las escuelas están adecuadamente modelados, no se encuentran diferencias sustantivas del rendimiento entre uno y otro tipo de gestión. Esta situación parecer ser producto del incremento masivo de la matrícula privada, que oferta una educación de bajo costo para familias de escasos recursos. Como consecuencia, se genera una gran heterogeneidad del sector privado, tanto en términos de calidad como en resultados de aprendizaje.

En el Anexo 3, se presenta los promedios de las pruebas a nivel de regiones y según la gestión, los que ilustran las diferencias regionales. Así, los datos muestran regiones donde existen brechas importantes entre las escuelas estatales versus las no estatales. En Matemáticas, la diferencia va desde 5 puntos en el Callao hasta más de 100 puntos en Ayacucho, Huancavelica, Huánuco y Apurímac ${ }^{8}$. En Lectura, se observa aún mayores diferencias que en Matemáticas y se agrega a Áncash a la lista. En Ciudadanía, aparece el mismo escenario de diferencias para las regiones citadas. Cabe destacar que las menores diferencias por gestión entre todas las competencias evaluadas ocurren en el Callao. La importante segregación en el país no es novedad (Benavides et al., 2014).

A partir de ello, en primer lugar, se confirma que los mayores puntajes en todas las competencias y las regiones sin excepción se encuentran en las escuelas de gestión privada. Tal vez, este no sea el caso a nivel distrital, como se ha encontrado en otras investigaciones. En segundo lugar, las diferencias intra e interregionales tienen un rango muy amplio, que ilustran la alta heterogeneidad de la diferenciación social, pero quizás también son consecuencia de una fuerte dispersión en la asignación y utilización de recursos humanos, físicos y financieros. Esto no significa necesariamente que frecuentar una escuela pública conduce a menores rendimientos: regiones como Tacna y Moquegua, por ejemplo, se distinguen por sus altos logros y $80 \%$ de sus alumnos asisten a escuelas públicas. 


\section{Conclusiones}

La encuesta muestral de 2013 permite medir la importancia de variables, sociales como el índice socioeconómico del alumno y de la escuela, como factores importantes en la determinación del rendimiento académico. En este sentido, estimar su impacto deviene crítico para contribuir al diseño y orientación de las políticas de mejoramiento de los aprendizajes.

Los reportes oficiales de la evaluación UMC-EM2013 presentan ampliamente, a través de modelos, los factores asociados que pueden influir sobre el rendimiento académico. De este modo, permiten espacio suficiente para investigar diversos aspectos de la desigualdad social en la educación primaria. Los resultados, como es usual, confirman una tendencia al crecimiento continuo y positivo del rendimiento conforme la condición social - medida por el índice social- es más alta. Esta relación se manifiesta como válida para las tres competencias medidas por la encuesta.

La fuerza de la influencia de la condición social se manifiesta claramente en la varianza explicada del rendimiento a través del llamado modelo multinivel nulo y mixto. En el modelo nulo, cuando ningún factor en particular interviene en la determinación del rendimiento, aproximadamente la mitad de la variabilidad de los logros académicos en cada una de las competencias es atribuible a las características propias de la escuela; y la otra mitad, atribuible a las características del alumno. Por construcción, estas características no son observadas por la encuesta y engloban todas aquellas que pueden perfilar al alumno y a la escuela. Cuando interviene el índice social en el modelo mixto, esta variabilidad al interior de las escuelas disminuye según la competencia: $39.9 \%$ en Matemáticas, 51.4 \% en Lectura y $34.4 \%$ en Ciudadanía. Estos valores son importantes y resaltan claramente la importancia del impacto del solo factor social por su alto poder de mediación.

Basándose en el índice social, el análisis cruzado de alumnos y escuelas entre categorías favorecidas y desfavorecidas permite apreciar los efectos de composición social. En efecto, los alumnos socialmente favorecidos asisten mayoritariamente (más de $70 \%$ de las veces) a escuelas también favorecidas. Ocurre lo mismo con los alumnos desfavorecidos, quienes también en proporción mayoritaria frecuentan las escuelas desfavorecidas. Esta distribución social se alinea igualmente con los logros académicos: se otorga mayores puntajes en todas las competencias a los alumnos socialmente favorecidos. Paradójicamente, aunque ocurre raramente (menos del 1\%), los alumnos desfavorecidos se benefician más si asisten a escuelas favorecidas.

Los acápites dedicados al análisis de logros por regiones aportan importantes lecciones. En efecto, muchas veces los promedios nacionales eclipsan realidades locales alarmantes. Así, 10 de las 26 regiones del país alcanzan el promedio nacional en cada una de las competencias. Además, aunque las diferencias pueden existir naturalmente entre regiones, los análisis muestran diferencias muy importantes en los logros de cada competencia tanto al interior de 
la misma región como entre las regiones. Esta dispersión interregional puede llegar a 150 puntos de diferencia con otra región y a 50 puntos al interior de una misma región. Cuando se perfila las regiones por la composición social de las escuelas, la dispersión es igualmente alarmante. En el país, coexisten regiones socialmente desfavorecidas que atiende a más de $60 \%$ de sus alumnos en esta categoría social frente a otras regiones que tienen menos del 5\% de alumnos en esta categoría. Los puntajes académicos traducen estas diferencias sociales regionales.

También, existen casos de fuerte contraste en la dependencia del índice social sobre el rendimiento escolar que atestiguan, por una parte, la poca equidad en la distribución del índice social y, por otra, el riesgo de efectos diferenciados que podrían obtenerse con una política social dirigida de manera uniforme a las familias de los alumnos sin distinguir su pertenencia regional. Así, hay regiones con alto rendimiento cuya pendiente tiene un valor de aproximadamente 20 puntos y regiones que, con un valor similar de pendiente, obtienen resultados extremadamente bajos, que llegan al extremo de hasta 150 puntos de diferencia en la misma competencia.

Sin profundizar en el debate recurrente sobre la diferencia de rendimiento entre escuelas estatales y no estatales, a nivel de regiones, este trabajo puede aportar a la discusión mostrando que los rendimientos en favor de las escuelas de gestión no estatal son siempre mayores que aquellos de las escuelas estatales (Anexo 3).

Los análisis presentados no eximen de dejar constancia sobre la cautela que debe acompañar las interpretaciones de estos resultados, pues se reitera el carácter muestral de sección cruzada de los datos, cuyos límites de causalidad son menores frente a encuestas longitudinales y experimentales. Asimismo, el índice socioeconómico utilizado tiene una utilidad temporal, puesto que, en tanto constructo multidimensional, posee componentes que probablemente evolucionarán en el futuro cuando aparezca nuevos índices.

Para futuras investigaciones, queda pendiente investigar la asociación entre la composición social de las escuelas con los procesos pedagógicos, psicosociales y psicológicos subyacentes a alumnos y docentes en el proceso de aprendizaje. Del mismo modo, queda por incorporar al análisis las mediaciones del rol parental, el capital cultural de la familia, los aspectos socioafectivos y emocionales de los aprendizajes, las aspiraciones de los alumnos, el clima escolar, entre otras canteras de interés que están muy contextualizados socialmente.

En este trabajo, se evita establecer relaciones de causa y efecto entre los factores que afectan los rendimientos escolares. Se propone, más bien, aportar pistas de reflexión suficientemente consistentes y significativas para ayudar a concebir políticas de educación justas y eficientes. Los resultados sugieren la aplicación de políticas sociales y educativas diferenciales de recursos a distintas escuelas que atienden alumnos diferentes y en contextos sociales también diferentes. Este parece ser un requisito para resolver el desafío de elevar el rendimiento de los alumnos y, al mismo tiempo, reducir las desigualdades asociadas 
a la condición social. No se puede ignorar que los resultados escolares son consecuencias históricas de políticas de educación, las que, a su vez, son producto de un proceso histórico y social propio.

Abatir la desigualdad en los aprendizajes basada en la condición social de alumnos y escuelas es una política social diferenciada y debe convertirse en una política emblemática de la más alta importancia. La educación es un proceso cuya transformación implica una visión de largo plazo y el futuro se construye desde ahora. Por este motivo, eliminar las desigualdades sociales en los aprendizajes desde la educación primaria deviene una cuestión urgente de política educativa. 


\section{Referencias bibliográficas}

Balarin, M. (2015). Las múltiples formas y efectos de la participación del sector privado en la educación. Proyecto Forge. Lima.

Benavides, M., León, J. y Etesse, M. (2014). Desigualdades educativas y segregación en el sistema educativo peruano. Una mirada comparativa en las pruebas Pisa 2000 y 2009. Lima: Grade.

Bourdieu, P. et Passeron, J. C. (1970). La reproduction. Elements pour une theorie du système d'enseignement. Paris: Les Editions de Minuit.

Calero, J., Escardíbul, O., Waisgrais, S. y Mediavilla, M. (2007). Desigualdades socioeconómicas en el sistema educativo español. Madrid: Ministerio de Educación.

Cuenca, R. (2013). La escuela pública en Lima Metropolitana. ¿Una institución en extinción? Revista Peruana de Investigación Educativa, (5), pp. 73-98.

Coleman, J. S., Campbell, E. Q., Hobson, C. J., McPartland, J., Mood, A. M., Weinfeld, F. D., and York, R. L. (1966). Equality and educational opportunity. Washington, DC: National Center for Educational Statistics, Office of Education, U.S. Department of Health, Education, and Welfare.

Caro, D. H. y Lenkeit, J. (2012). An analytical approach to study educational inequalities: 10 hypothesis tests in PIRLS 2006. International Journal of Research \& Method in Education, 35(1), 3-30.

Consejo Nacional de Educación (2007). Proyecto educativo nacional al 2021: La educación que queremos. Lima: Consejo Nacional de Educación.

Cueto, S. (2007). Las evaluaciones nacionales e internacionales de rendimiento escolar en el Perú: Balance y perspectivas. En Grupo de Análisis para el Desarrollo (Ed.), Investigación, políticas y desarrollo en el Perú (pp. 405455). Lima: Grupo de Análisis para el Desarrollo.

Cueto, S., León, J.y Miranda, A. (2014). La composición de la escuela y su asociación con el rendimiento y características socioemocionales de los estudiantes. Niños del Milenio. Recuperado de <www.ninosdelmilenio.org >

Guadalupe, C. y Castillo, L. (2014). Diferencias regionales en políticas de apoyo al aprendizaje y su posible impacto sobre los niveles de logro (documento de Discusión). Lima, Centro de Investigación de la Universidad del Pacífico.

Guadalupe, C. y Villanueva, A. (2014). PISA 2009/2000 en América Latina: Una relectura de los cambios en el desempeño lector y su relación con las condiciones sociales. Apuntes: Revista de Ciencias Sociales, 40(72), 157192.

Hanushek, E., y Woessman, L. (2011). The economics of international differences in educational achievement. In Hanushek E., Machin S. y Woessmann, L. (Eds.), Handbookof Economics of Education. Vol 3. The Economics of Education (pp. 89-200). Amsterdam: North Holland. 
León, J. y Min-Jong Youn. (2016). El efecto de los procesos escolares en el rendimiento en Matemática y las brechas de rendimiento debido a diferencias socioeconómicas de los estudiantes peruanos. Revista Peruana de Investigación Educativa, (8), 149-180.

León, J. y Collahua, Y. (2016). El efecto del nivel socioeconómico en el rendimiento de los estudiantes peruanos: Un balance de su efecto en los últimos quince años. Investigación para el desarrollo en el Perú: Once balances. Lima: Grade.

Ministerio de Educación del Perú - Unidad de Medición de la Calidad de los Aprendizajes (2006). Evaluación nacional del rendimiento estudiantil 2004. ¿Cómo disminuir la inequidad del sistema educativo peruano y mejorar el rendimiento de sus estudiantes? Factores explicativos más relevantes en la Evaluación Nacional 2004. Lima: Ministerio de Educación.

¿Cuánto aprenden nuestros estudiantes al término de la educación primaria? Informe de logros de aprendizaje y sus factores asociados en la Evaluación Muestral 2013. Lima: Ministerio de Educación.

(2015). Reporte técnico de la Evaluación Muestral de estudiantes de $6^{\circ}$ de primaria 2013. Lima: Ministerio de Educación.

(2016). Regiones en perspectiva: la influencia de los factores asociados al aprendizaje al término de la educación primaria. Estudios Breves 2. Lima: Ministerio de Educación.

Miranda, L. (2008). Factores asociados al rendimiento escolar y sus implicancias para la política educativa del Perú. En M. Benavides (Ed.), Análisis de programas, procesos y resultados educativos en el Perú: contribuciones empíricas para el debate (pp. 11-39). Lima: Grupo de Análisis para el Desarrollo.

Monseur, C. et Crahay, M (2008). Composition académique et sociale des établissements, efficacité et inégalités scolaires: Une comparaison internationale. Revue Francaise de Pédagogie, (164), Julio-Septiembre.

Muelle, L. (2016). Factores de riesgo en el bajo desempeño académico y desigualdad social en el Perú según PISA 2012. Revista Apuntes, 43(79), $10-45$.

Organization for Economic Co-operation and Development [OECD] (2014). PISA 2012 Results: What students know and can do. Student Performance in Mathematics, Reading and Science, Volume I. Paris: OECD Publishing.

Rumberger R., and Palardy G, (2005). Does segregation still matter? The impact of student composition on academic achievement in High School. Teachers College Record, 107(9), 1999-2045.

Sirin, S. R. (2005). Socioeconomic status and academic achievement: A metaanalytic review of research. Review of Educational Research, 75(3), 417453. 
Snijders T., and Bosker R. (2012). Multilevel analysis: An introduction to basic and advanced Multilevel Modeling. ${ }^{\text {nd }}$ Ed. London: Sage Publishers.

Unesco-Orealc (2016). Terce - Factores asociados. Cuadernillo $N^{\circ}$ 3. Laboratorio Latinoamericano de la evaluación de la calidad de la educación. Santiago de Chile: Unesco.

Willms, J. D. (2006). Las brechas de aprendizaje: Diez preguntas de la política educativa a seguir en relación con el desempeño y la equidad en las escuelas $y$ los sistemas educativos. Montreal: Instituto de Estadística de la Unesco. 
Anexo 1.

Distribución de promedios y errores estándares según Matemáticas (M), Lectura (L) y Ciudadanía (C), e índices socioeconómicos del alumno y de la escuela, según regiones

\begin{tabular}{|c|c|c|c|c|c|c|c|c|c|c|}
\hline Región & M & ee & $\mathrm{L}$ & ee & $\mathrm{Ca}$ & ee & ISE & e.e & $\begin{array}{c}\text { ISE- } \\
\text { escuela }\end{array}$ & e.e \\
\hline Amazonas & 478.4 & $(5.26)$ & 473.1 & $(4.78)$ & 479.4 & $(5.11)$ & -0.767 & 0.05 & -0.764 & 0.05 \\
\hline Áncash & 476.7 & $(5.36)$ & 476.2 & $(5.01)$ & 475.8 & $(4.79)$ & -0.210 & 0.05 & -0.213 & 0.05 \\
\hline Apurímac & 436.8 & $(5.60)$ & 431.4 & (6.14) & 440.1 & $(5.82)$ & -0.596 & 0.06 & -0.609 & 0.06 \\
\hline Arequipa & 553.0 & (3.91) & 547.2 & $(3.42)$ & 542.1 & (3.31) & 0.569 & 0.04 & 0.566 & 0.04 \\
\hline Ayacucho & 463.1 & $(7.34)$ & 458.7 & (7.07) & 463.6 & (6.98) & -0.362 & 0.07 & -0.378 & 0.08 \\
\hline Cajamarca & 480.7 & $(9.48)$ & 459.6 & (7.08) & 461.0 & $(7.46)$ & -0.866 & 0.09 & -0.869 & 0.09 \\
\hline Callao & 540.5 & (3.613) & 549.9 & $(2.953)$ & 543.3 & $(3.430)$ & 0.618 & 0.04 & 0.626 & 0.04 \\
\hline Cusco & 479.0 & $(5.437)$ & 467.8 & $(5.392)$ & 472.1 & $(4.848)$ & -0.355 & 0.05 & -0.364 & 0.05 \\
\hline Huancavelica & 450.6 & $(5.438)$ & 422.9 & $(5.444)$ & 427.4 & $(5.420)$ & -0.849 & 0.06 & -0.893 & 0.06 \\
\hline Huánuco & 447.2 & $(5.300)$ & 440.7 & $(6.012)$ & 445.7 & (5.295) & -0.709 & 0.06 & -0.710 & 0.06 \\
\hline Ica & 520.6 & $(5.602)$ & 521.1 & (3.907) & 522.1 & $(4.222)$ & 0.422 & 0.03 & 0.428 & 0.03 \\
\hline Junín & 518.3 & $(6.046)$ & 506.9 & $(4.803)$ & 508.7 & $(4.786)$ & -0.025 & 0.06 & -0.029 & 0.06 \\
\hline La Libertad & 491.1 & $(4.950)$ & 490.1 & $(4.874)$ & 490.8 & $(4.941)$ & -0.308 & 0.06 & -0.325 & 0.06 \\
\hline Lambayeque & 507.8 & $(4.681)$ & 510.6 & $(4.455)$ & 515.9 & $(4.472)$ & 0.018 & 0.05 & -0.003 & 0.06 \\
\hline $\begin{array}{l}\text { Lima } \\
\text { Metropolitana }\end{array}$ & 542.6 & $(4.590)$ & 550.7 & (3.981) & 543.0 & $(4.143)$ & 0.770 & 0.04 & 0.783 & 0.04 \\
\hline $\begin{array}{l}\text { Lima } \\
\text { Provincias }\end{array}$ & 515.7 & (3.748) & 520.1 & (4.323) & 519.6 & $(4.314)$ & 0.239 & 0.05 & 0.244 & 0.05 \\
\hline Loreto & 411.6 & (4.909) & 424.4 & (6.129) & 439.8 & $(5.340)$ & -0.66 & 0.07 & -0.691 & 0.07 \\
\hline Madre de Dios & 474.7 & $(5.843)$ & 488.8 & $(5.801)$ & 483.6 & $(6.166)$ & 0.054 & 0.05 & 0.057 & 0.05 \\
\hline Moquegua & 554.9 & $(5.348)$ & 547.7 & $(4.867)$ & 537.6 & $(4.966)$ & 0.656 & 0.06 & 0.657 & 0.06 \\
\hline Pasco & 497.3 & $(6.221)$ & 489.9 & $(5.702)$ & 495.2 & $(5.023)$ & -0.17 & 0.07 & -0.187 & 0.07 \\
\hline Piura & 487.5 & $(3.480)$ & 489.2 & (3.555) & 501.7 & $(3.436)$ & -0.41 & 0.05 & -0.417 & 0.05 \\
\hline Puno & 460.4 & $(5.977)$ & 446.3 & $(5.471)$ & 449.2 & (5.947) & -0.31 & 0.08 & -0.320 & 0.08 \\
\hline San Martín & 464.9 & $(5.052)$ & 471.9 & $(4.696)$ & 480.1 & (4.585) & -0.46 & 0.07 & -0.469 & 0.07 \\
\hline Tacna & 566.2 & $(4.941)$ & 555.3 & $(3.826)$ & 549.7 & $(4.376)$ & 0.615 & 0.05 & 0.619 & 0.05 \\
\hline Tumbes & 473.8 & $(4.559)$ & 487.3 & $(4.177)$ & 489.7 & (3.863) & 0.145 & 0.06 & 0.140 & 0.06 \\
\hline Ucayali & 454.2 & $(4.443)$ & 474.0 & $(4.637)$ & 481.8 & (3.987) & -0.23 & 0.05 & -0.253 & 0.06 \\
\hline
\end{tabular}

N.B.: Todos los valores son significativos a $\mathrm{p}<0.001$.

Fuente: Elaboración propia a partir de la UMC-EM 2013 
Anexo 2.

Coeficientes $(\beta)$ y errores estándares (e.e) de los puntajes en Matemáticas (M), Lectura (L) y Ciudadanía de las regresiones multinivel del índice socioeconómico del alumno (ISE) según regiones

\begin{tabular}{|c|c|c|c|c|c|c|}
\hline Région & $\beta \mathrm{M}$ & e.e & $\beta \quad L$ & e.e & $\beta C$ & e.e \\
\hline Amazonas & 24.0 & 2.64 & 31.2 & 2.74 & 31.5 & 2.56 \\
\hline Áncash & 34.4 & 1.99 & 38.7 & 2.06 & 37.8 & 1.97 \\
\hline Apurímac & 22.6 & 2.15 & 28.7 & 2.32 & 30.8 & 2.33 \\
\hline Arequipa & 29.4 & 2.74 & 24.7 & 2.64 & 25.4 & 2.39 \\
\hline Ayacucho & 25.1 & 2.58 & 26.7 & 2.66 & 32.6 & 2.59 \\
\hline Cajamarca & 29.6 & 2.62 & 36.3 & 2.63 & 39.7 & 2.43 \\
\hline Callao & 22.3 & 2.56 & 21.4 & 2.63 & 25.6 & 2.36 \\
\hline Cusco & 26.6 & 1.97 & 32.6 & 1.98 & 33.4 & 1.92 \\
\hline Huancavelica & 14.8 & 2.76 & 21.4 & 2.84 & 23.2 & 2.75 \\
\hline Huánuco & 25.0 & 2.31 & 27.1 & 2.38 & 25.8 & 2.34 \\
\hline Ica & 23.8 & 2.31 & 25.7 & 2.33 & 30.2 & 2.21 \\
\hline Junín & 25.3 & 2.30 & 28.3 & 2.13 & 31.6 & 2.10 \\
\hline La Libertad & 23.2 & 2.00 & 28.7 & 2.08 & 30.3 & 1.92 \\
\hline Lambayeque & 23.2 & 1.98 & 25.7 & 2.14 & 28.1 & 1.99 \\
\hline $\begin{array}{l}\text { Lima } \\
\text { Metropolitana }\end{array}$ & 26.7 & 2.42 & 27.1 & 2.39 & 28.6 & 2.21 \\
\hline $\begin{array}{l}\text { Lima } \\
\text { Provincia }\end{array}$ & 28.2 & 2.67 & 32.2 & 2.67 & 33.5 & 2.48 \\
\hline Loreto & 16,6 & 2.29 & 18.5 & 2.50 & 20.9 & 2.62 \\
\hline Madre de Dios & 26,8 & 3.45 & 25.4 & 3.76 & 31.0 & 3.47 \\
\hline Moquegua & 26,9 & 3,61 & 22,9 & 3.49 & 26.9 & 3.14 \\
\hline Pasco & 37,9 & 2,87 & 37,7 & 2.87 & 37.0 & 2.66 \\
\hline Piura & 22,9 & 1,83 & 28,2 & 1.92 & 30.2 & 1.82 \\
\hline Puno & 31,3 & 2,48 & 31,6 & 2.56 & 34.6 & 2.45 \\
\hline San Martín & 24,6 & 2,07 & 32,8 & 2.06 & 32.8 & 1.95 \\
\hline Tacna & 22,0 & 2,75 & 20,0 & 2.62 & 21.5 & 2.30 \\
\hline Tumbes & 21,9 & 2,48 & 24,9 & 2.57 & 25.0 & 2.57 \\
\hline Ucayali & 21,0 & 2,22 & 22,8 & 2.36 & 25.1 & 2.34 \\
\hline
\end{tabular}

N.B: Todos los coeficientes de $\beta$ son significativos a $\mathrm{p}<0.001$.

Fuente: Elaboración propia a partir de la UMC-EM 2013 
Anexo 3.

Promedio y errores estándares (e.e) de Matemáticas (M), Lectura (L) y Ciudadanía (C) por gestión estatal (Pub.) y no estatal (Priv.), según regiones

\begin{tabular}{|c|c|c|c|c|c|c|c|c|c|c|c|c|}
\hline Reg & $\begin{array}{c}\mathrm{M} \\
\mathrm{Pub}\end{array}$ & e.e & $\begin{array}{c}\text { M } \\
\text { Priv }\end{array}$ & e.e & $\begin{array}{c}\mathrm{L} \\
\text { Pub }\end{array}$ & e.e & $\begin{array}{c}\mathrm{L} \\
\text { Priv }\end{array}$ & e.e & $\begin{array}{c}\text { C } \\
\text { Pub }\end{array}$ & e.e & $\begin{array}{c}\text { C } \\
\text { Priv }\end{array}$ & e.e \\
\hline Amazonas & 478.2 & 5.29 & 508.7 & 8.17 & 472.7 & 4.80 & 553.7 & 1.01 & 478.9 & 5.12 & 569.3 & 5.42 \\
\hline Áncash & 465.8 & 6.05 & 556.3 & 8.99 & 463.7 & 5.68 & 567.4 & 6.82 & 464.1 & 5.30 & 561.2 & 9.51 \\
\hline Apurímac & 432.7 & 5.85 & 535.8 & 12.21 & 426.8 & 6.44 & 544.9 & 4.16 & 437.1 & 6.10 & 511.8 & 8.52 \\
\hline Arequipa & 537.5 & 5.10 & 575.7 & 5.70 & 528.8 & 4.16 & 574.0 & 5.07 & 528.6 & 3.79 & 561.9 & 5.82 \\
\hline Ayacucho & 452.9 & 7.09 & 567.9 & 10.87 & 447.2 & 6.99 & 576.4 & 6.28 & 453.2 & 6.95 & 569.2 & 6.54 \\
\hline Cajamarca & 478.4 & 9.83 & 521.0 & 32.45 & 455.6 & 7.10 & 530.1 & 31.56 & 457.8 & 7.68 & 518.12 & 22.25 \\
\hline Callao & 538.7 & 5.08 & 544.0 & 4.30 & 543.2 & 3.83 & 562.1 & 4.60 & 542.3 & 4.31 & 545.1 & 5.73 \\
\hline Cusco & 468.6 & 6.08 & 552.4 & 12.36 & 455.5 & 6.13 & 554.4 & 9.12 & 461.8 & 5.43 & 545.5 & 11.28 \\
\hline Huancavelica & 450.0 & 5.47 & 555.3 & 9.04 & 422.2 & 5.47 & 548.0 & 17.71 & 426.8 & 5.45 & 544.7 & 8.21 \\
\hline Huánuco & 438.0 & 5.49 & 553.9 & 13.59 & 430.5 & 6.20 & 558.6 & 10.60 & 437.5 & 5.51 & 541.4 & 6.30 \\
\hline Ica & 510.1 & 7.23 & 553.6 & 7.77 & 508.8 & 5.00 & 559.8 & 6.16 & 512.8 & 5.24 & 551.2 & 7.70 \\
\hline Junín & 510.5 & 6.88 & 567.0 & 9.64 & 496.2 & 5.29 & 574.4 & 9.19 & 500.9 & 5.30 & 557.5 & 8.98 \\
\hline La Libertad & 476.2 & 5.59 & 554.0 & 8.47 & 472.1 & 5.35 & 565.8 & 6.66 & 474.4 & 5.64 & 559.9 & 6.18 \\
\hline Lambayeque & 493.0 & 5.40 & 554.7 & 8.76 & 493.1 & 4.66 & 565.8 & 9.46 & 502.9 & 4.86 & 556.9 & 9.59 \\
\hline $\begin{array}{l}\text { Lima } \\
\text { Metropolitana }\end{array}$ & 526.9 & 7.67 & 561.1 & 5.44 & 533.5 & 6.77 & 571.0 & 4.64 & 533.9 & 6.76 & 553.6 & 4.86 \\
\hline $\begin{array}{l}\text { Lima } \\
\text { Provincia }\end{array}$ & 504.1 & 3.98 & 55 & 6.49 & 50 & 4.35 & 5 & 6.96 & 50 & 5.01 & 555.9 & 6.33 \\
\hline Loreto & 407.2 & 4.67 & 470.4 & 30.16 & 420.0 & 5.81 & 484.3 & 40.63 & 436.4 & 4.90 & 486.43 & 38.40 \\
\hline Madre de Dios & 472.3 & 6.07 & 493.8 & 16.41 & 484.2 & 5.80 & 525.9 & 16.87 & 482.1 & 6.27 & 495 & 1.80 \\
\hline Moquegua & 548.3 & 6.08 & 581.6 & 11.25 & 538.7 & 5.72 & 583.6 & 6.61 & 530.7 & 5.82 & 565.8 & 6.78 \\
\hline Pasco & 491.0 & 6.09 & 591.0 & 26.50 & 485.6 & 6.00 & 554.0 & 13.70 & 491.1 & 5.26 & 554.9 & 12.01 \\
\hline Piura & 475.8 & 3.90 & 546.9 & 6.62 & 474.5 & 4.03 & 563.5 & 5.47 & 490.5 & 4.03 & 558.4 & 4.03 \\
\hline Puno & 454.0 & 6.66 & 502.4 & 12.03 & 437.3 & 6.13 & 505.3 & 10.53 & 443.3 & 6.79 & 488.3 & 9.26 \\
\hline San Martín & 462.2 & 5.18 & 528.8 & 3.17 & 468.9 & 4.73 & 544.4 & 10.24 & 478.7 & 4.75 & 512.5 & 12.34 \\
\hline Tacna & 557.2 & 5.55 & 594.6 & 10.09 & 545.4 & 4.51 & 586.6 & 6.57 & 543.5 & 5.10 & 569.5 & 8.07 \\
\hline Tumbes & 466.9 & 5.14 & 525.4 & 7.64 & 478.3 & 4.77 & 555.0 & 6.54 & 483.4 & 4.30 & 536.7 & 7.85 \\
\hline Ucayali & 449.8 & 4.74 & 531.5 & 4.59 & 470.2 & 4.93 & 539.3 & 8.32 & 478.4 & 4.20 & 541.4 & 9.82 \\
\hline
\end{tabular}

N.B.: Todos los valores son significativos a $\mathrm{p}<0.001$.

Fuente: Elaboración propia a partir de la UMC-EM 2013 